\title{
The CMS "Higgs Boson Goose Game"
}

\author{
Francesca R. Cavallo*广 \\ $\operatorname{INFN}(I T)$ \\ E-mail: francesca.cavallo@bo.infn.it

\section{Marco Dallavalle} \\ INFN (IT) \\ E-mail: marco.dallavalle@bo.infn.it
}

Building and operating the CMS Detector is a complicated endeavour! Now, more than 20 years after the detector was conceived, the CMS Bologna group proposes to follow the steps of this challenging project by playing "The Higgs Boson Goose Game", illustrating CMS activities and goals.

The concept of the game is inspired by the traditional "Game of the Goose". The underlying idea is that the progress of building and operating a detector at the LHC is similar to the progress of the pawns on the game board: it is fast at times, bringing rewards and satisfaction, while sometimes unexpected problems cause delays or even a step back requiring CMS scientists to use all of their skill and creativity to devise new solutions.

The European Physical Society Conference on High Energy Physics

22-29 July 2015

Vienna, Austria

\footnotetext{
* Speaker.

${ }^{\dagger}$ Thanks to Alberto Monti for the original drawings and to Marzena Lapka for technical support.
} 


\section{Introduction}

CMS is one of the four major particle detectors at CERN's Large Hadron Collider (LHC). Its name stands for Compact Muon Solenoid: Compact because it is "small" with respect to its enormous weight, Muon for one of the types of particle that it can detect, and Solenoid for the coil that forms its huge superconducting magnet. While playing this game, one follows the story of the CMS Experiment from assembly of the detector to discovery of the Higgs boson. The concept of the game was developed from an idea proposed by Alberto Monti for a CMS poster, consisting of a multi-picture frame of the CMS detector inspired by boards for the Game of the Goose. Soon, within the CMS Bologna group, it became clear that the concept could be developed into a real board game, telling the CMS history from detector construction to the first great discovery, through good and bad times.

Alberto Monti is the author of the drawings and of the board frame. The board spaces and their captions were studied and written (with great fun) by the CMS Bologna group.

Our aim is that people learn something interesting about particle physics while having fun with this game.

\section{The concept}

The game, whose board is shown in Fig. 1, is inspired by the traditional "Game of the goose".

The underlying idea is that the progress of an experiment is similar to the progress of the pawns on the game board: it is fast at moments, bringing rewards and satisfaction, while sometimes unexpected problems impose delays or even some steps back: physicists must then use all of their skills and creativeness to devise new solutions.

The project tells the story of the CMS experiment from integration to the Higgs discovery.

The sequence of steps features pictures of the detector, of the control room, of computer screen-shots etc, depending on the meaning of the single squares within the context of the game development.

Some of the squares show instead traditional and symbolic drawings, inspired by real old goose game boards, that have been re-interpreted in the captions and adapted to the CMS and CERN context.

In the bottom-right corner a historical picture reminds of CERN foundation in 1954, suggesting that this was indeed the true beginning of the story.

The goal of the path is of course the Higgs discovery!

The game can be played by up to six players, each pawn bearing the symbol of one of the six quarks.

In the game box a booklet is included that reports, on top of the basic instructions, a CMS chronology that helps the players understanding the meaning of individual steps, and a brief history of the Game of the Goose from ancient Egypt to XIX century.

\section{Example squares}

We describe here a few examples of game steps in order to illustrate the spirit of the project. 


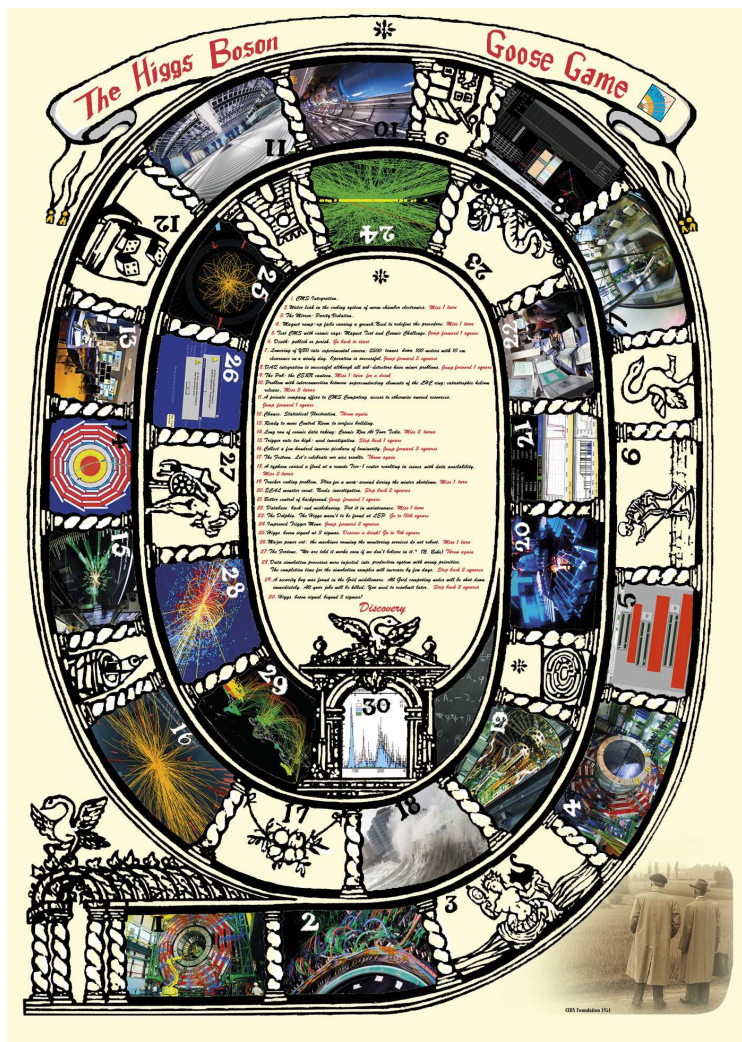

Figure 1: The board of "The CMS Game of the Goose"

\subsection{Steps referring to real events}

By 2007 most of the CMS detector was assembled and integrated in a surface building [1]. This is the start of the game path: "1. CMS Integration". See Fig. 2

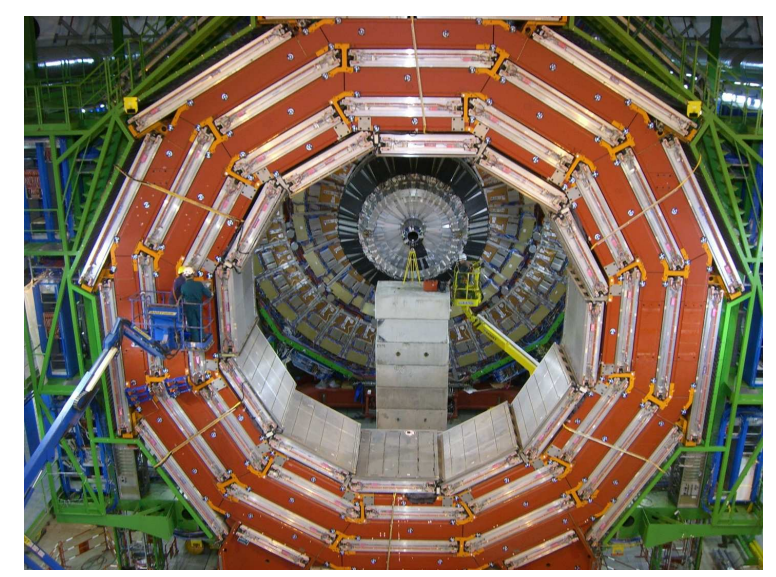

Figure 2: A picture of the CMS detector during assembling in the surface hall.

The lowering of the 2000 tonnes central barrel slice, known as YB0, was a spectacular event attracting journalists from all over the world. [2] Within the game this event is referred to in the 
step: "7. Lowering of YB0 into experimental cavern: 2000 tonnes down 100 meters with $10 \mathrm{~cm}$ clearance on a windy day. Operation is successful. Jump forward 5 squares." See Fig.3

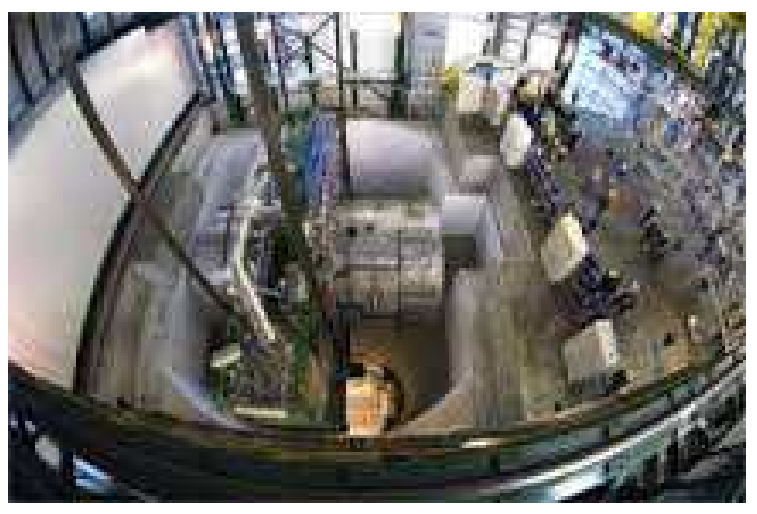

Figure 3: A snapshot of the historical lowering of YB0 into the experimental cavern.

On September 10, 2008 the LHC started successful injection of the first low-energy circulating proton beam. Nine days later, an incident caused a massive helium leak that damaged some of the magnets [3]. The LHC was stopped for one year. You may find this incident in the step: "10. Problem with interconnection between superconducting elements of the LHC ring: catastrophic helium release. Miss 4 turns." See Fig. 4

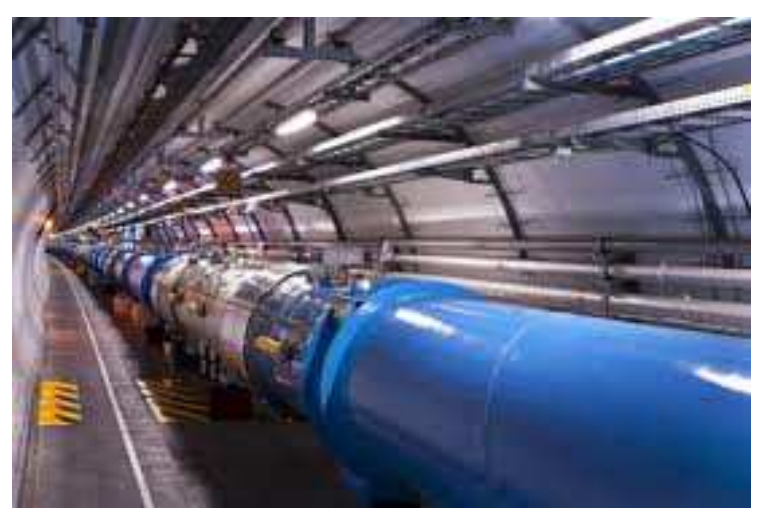

Figure 4: A view of the LHC tunnel and the superconducting magnets.

\subsection{Steps featuring traditional drawings}

We report here some examples of traditional squares, with their interpretation in the CERN context:

“9. The Pub: the CERN canteen. Miss 1 turn for a drink." See Fig. 5 left.

In the following path this square is mentioned again in the step:

“25. Higgs boson signal at 3 sigmas. Deserve a drink! Go to 9th square.” See Fig. 5 right.

Other examples are:

"12. The Chance. Statistical fluctuation. Throw again." See Fig. 6 left.

"27. The Fortune. "We are told it works even if we don t believe in it." (N.Bohr) Throw again." See Fig. 6 right. 

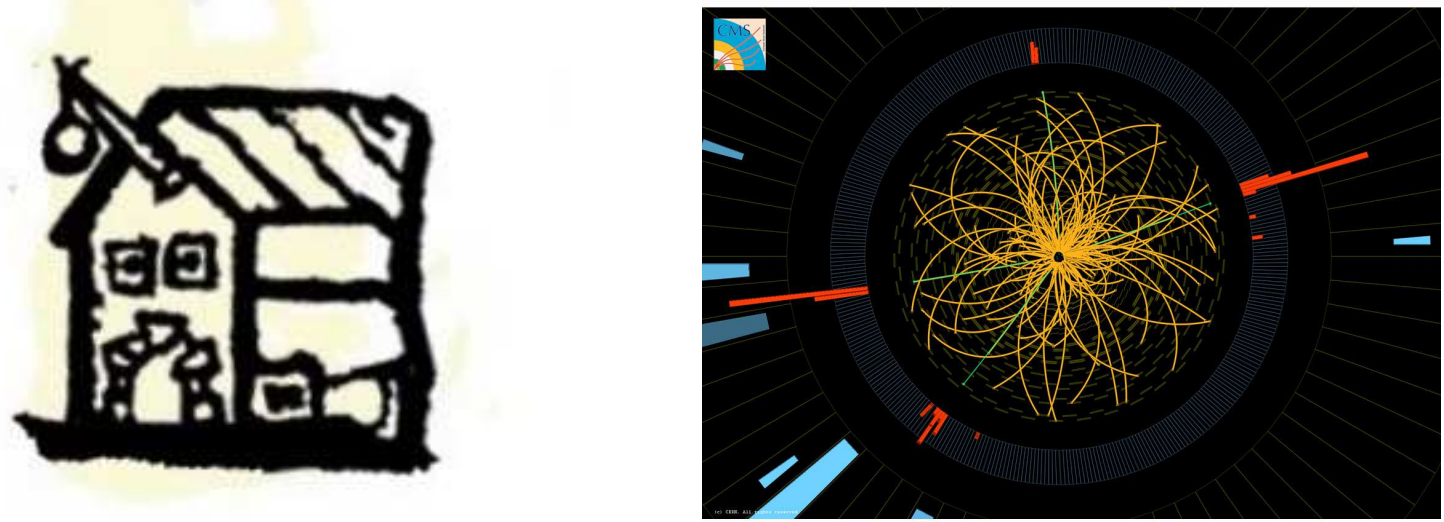

Figure 5: Left: original drawing inspired to the old symbol of the Tavern. Right: event display of a candidate Higgs decay. The hint of a possible discovery deserves to be celebrated with a drink!
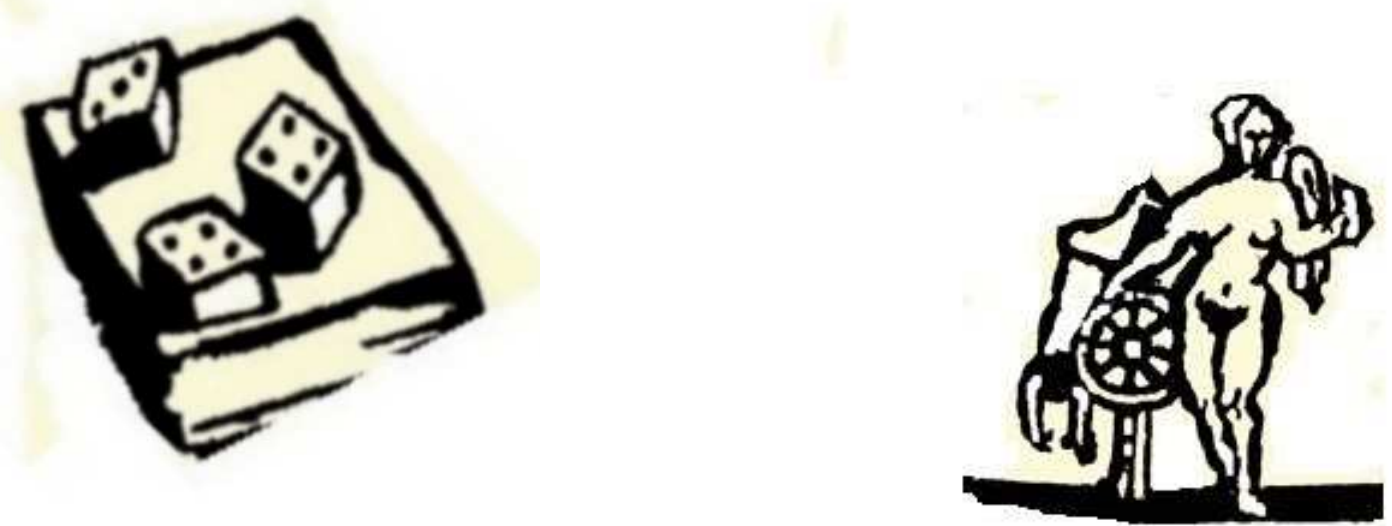

Figure 6: Original drawings representing the Chance and the Fortune

\subsection{The finish line}

Finally, on 4 July 2012 the announcement was given of the discovery of a new particle by CMS and ATLAS [4]. This is the finish line of the game and its last square:

“30. Higgs boson signal beyond 5 sigmas!” See Fig. 7 


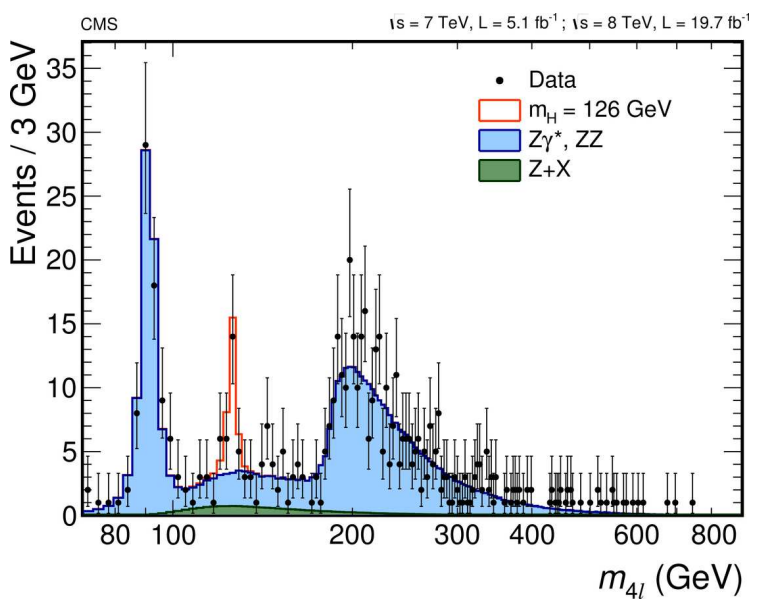

Figure 7: Final result published by the CMS collaboration, showing the signal of Higgs boson decaying into four leptons. [5]

\section{Conclusions}

This game is presently available at http://cms.web.cern.ch/news/cms-shop. It is a CMS outreach and educational tool: we hope many people will enjoy playing and learn something interesting about particle physics experiments.

\section{References}

[1] The CMS Collaboration, The CMS experiment at the CERN LHC, 2008 JINST 3 S08004.

[2] See for instance the BBC report: http://news.bbc.co.uk/2/hi/science/nature/6402493.stm.

[3] G. Brumfiel, Eight-month delay for LHC, Nature 455, 1015 (2008).

[4] The CMS Collaboration, Observation of a new boson at a mass of $125 \mathrm{GeV}$ with the CMS experiment at the LHC, Physics Letters B 716 (2012) 30-61.

[5] The CMS Collaboration, Measurement of the properties of a Higgs boson in the four-lepton final state, Phys. Rev. D 89 (2014) 092007. 